\title{
A role for vitamin $D$ and omega-3 fatty acids in major depression? An exploration using genomics
}

\author{
Yuri Milaneschi', Wouter J. Peyrot ${ }^{1}$, Michel G. Nivard $\mathbb{D}^{2}$, Hamdi Mbarek $\mathbb{D}^{2,3}$, Dorret I. Boomsma $\mathbb{C}^{2}$ and \\ Brenda W.J.H. Penninx ${ }^{1}$
}

\begin{abstract}
Trials testing the effect of vitamin D or omega-3 polyunsaturated fatty acid (n3-PUFA) supplementation on major depressive disorder (MDD) reported conflicting findings. These trials were inspired by epidemiological evidence suggesting an inverse association of circulating 25-hydroxyvitamin D (25-OH-D) and n3-PUFA levels with MDD. Observational associations may emerge from unresolved confounding, shared genetic risk, or direct causal relationships. We explored the nature of these associations exploiting data and statistical tools from genomics. Results from genome-wide association studies on 25-OH-D ( $N=79366)$, n3-PUFA ( $N=24925)$, and MDD (135 458 cases, 344901 controls) were applied to individual-level data (>2000 subjects with measures of genotype, DSM-IV (Diagnostic and Statistical Manual of Mental Disorders, 4th edition) lifetime MDD diagnoses and circulating 25-OH-D and n3-PUFA) and summary-level data analyses. Shared genetic risk between traits was tested by polygenic risk scores (PRS). Two-sample Mendelian Randomization (2SMR) analyses tested the potential bidirectional causality between traits. In individual-level data analyses, PRS were associated with the phenotype of the same trait (PRS 25-OH-D $p=$ 1.4e -20 , PRS n3-PUFA $p=9.3 e-6$, PRS MDD $p=1.4 \mathrm{e}-4$ ), but not with the other phenotypes, suggesting a lack of shared genetic effects. In summary-level data analyses, $2 S M R$ analyses provided no evidence of a causal role on MDD of 25-OH-D ( $p=0.50)$ or n3-PUFA ( $p=0.16)$, or for a causal role of MDD on 25-OH-D $(p=0.25)$ or n3-PUFA $(p=0.66)$. Applying genomics tools indicated that shared genetic risk or direct causality between $25-\mathrm{OH}-\mathrm{D}, \mathrm{n3}-\mathrm{PUFA}$, and MDD is unlikely: unresolved confounding may explain the associations reported in observational studies. These findings represent a cautionary tale for testing supplementation of these compounds in preventing or treating MDD.
\end{abstract}

\section{Introduction}

In recent years a growing interest has emerged in the potential value of nutritional supplementation in mood disorders. Large-scale randomized controlled trials such as MoodFOOD ${ }^{1}(N \sim 1000)$ or VITAL-DEP ${ }^{2}(N \sim 25000)$ have been established to test the effect of supplementation in the prevention of depression. The rationale for these interventions was inspired from epidemiological evidence of an inverse association between depression and

\footnotetext{
Correspondence: Yuri Milaneschi (y.milaneschi@ggzingeest.nl)

${ }^{1}$ Department of Psychiatry, Amsterdam Public Health and Amsterdam

Neuroscience, Amsterdam UMC, Vrije Universiteit/GGZ inGeest, Amsterdam, Netherlands

²Department of Biological Psychology, VU University Amsterdam, Amsterdam, Netherlands

Full list of author information is available at the end of the article.
}

circulating concentrations of compounds that could be easily supplemented, such as vitamin D and omega-3 polyunsaturated fatty acids (n3-PUFA). Meta-analyses of large observational studies consistently reported crosssectional, and to a lesser extent longitudinal, associations of 25-hydroxyvitamin-D (25-OH-D, the body reserve of vitamin D) and n3-PUFA with depression. ${ }^{3-6}$ In $>2000$ psychiatrically well-characterized participants from the Netherlands Study of Depression and Anxiety (NESDA), we previously confirmed ${ }^{7,8}$ lower concentrations of 25$\mathrm{OH}-\mathrm{D}$ and n3-PUFA in patients with depression as compared to healthy controls, and inverse associations with symptom severity. Previous clinical studies attempted to translate this observational evidence in interventions: meta-analyses ${ }^{9-12}$ pooling results from trials testing

\section{(c) The Author(s) 2019}

(c) (i) Open Access This article is licensed under a Creative Commons Attribution 4.0 International License, which permits use, sharing, adaptation, distribution and reproduction in any medium or format, as long as you give appropriate credit to the original author(s) and the source, provide a link to the Creative Commons license, and indicate if changes were made. The images or other third party material in this article are included in the article's Creative Commons license, unless indicated otherwise in a credit line to the material. If material is not included in the article's Creative Commons license and your intended use is not permitted by statutory regulation or exceeds the permitted use, you will need to obtain permission directly from the copyright holder. To view a copy of this license, visit http://creativecommons.org/licenses/by/4.0/. 
the effect of vitamin D and omega-3 supplements on depression reported conflicting findings, with studies showing both small clinical effect and no effect. Conflicting findings have been commonly attributed to heterogeneity and limitations in study design, including small samples, inadequacy of supplementation dose, or lack of blood markers to trace the biological availability of the compounds supplemented. As a consequence, the implementation of larger and methodologically robust trials has been repeatedly advocated to overcome this impasse.

Before moving to further intervention studies it is however crucial to take a step back and to obtain a clearer knowledge of the exact nature of the observational associations that inspired the supplementation interventions. Observational associations could emerge indeed from different scenarios. In the first of these scenarios, observational associations emerging in nutritional epidemiology may be the product of unresolved confounding, as recently highlighted by Ioannidis, ${ }^{13}$ since almost all nutritional variables are correlated with one another and with many social and behavioral factors. In the second scenario, an observational association may also be detected when both traits are epiphenomena stemming from the same etiological root, sharing part of their genetic liability. Previous studies ${ }^{14,15}$ reported non-significant genome-wide genetic correlations between MDD and 25-OH-D or n3-PUFA. Nevertheless, these results may have been affected by the different genetic architectures of MDD (highly polygenic with many genetic variants with small effects) and 25-OH-D or n3-PUFA (few biologically relevant loci with relatively larger effect sizes). For instance, genome-wide significant loci for $25-\mathrm{OH}-\mathrm{D}^{14}$ harbor relevant genes for its synthesis and metabolism such as GC (vitamin D binding protein, transporting vitamin D to target tissue), DHCR7 (7-dehydrocholesterol reductase, involved in vitamin D synthesis), and CYP2R1 and CYP24A1 (both cytrochrome P450 family enzymes, catalyzing the transformation of vitamin $\mathrm{D}$ in its active form). In the third scenario, the most favorable for clinical translation, an observational association is detected because a trait (25-OH-D/n3-PUFA) is effectively causal for another (MDD). For vitamin D, hypotheses based on preclinical data suggested a potential impact of vitamin $\mathrm{D}$ on brain structure (vitamin D receptor is expressed in prefrontal cortex, amygdala, and hippocampus) and pathophysiological processes relevant for mood. ${ }^{16}$ Similarly, n3-PUFA may exert important biological effects in the nervous system, modulating membrane fluidity, serotonergic transmission, and inflammatory response. ${ }^{17}$ Finally, the fourth scenario involves reverse causation, since depression may impact on 25-OH-D and n3-PUFA levels via habits related, for instance, to sunlight exposure or diet. $^{18}$
It is not trivial to disentangle from which of the above scenarios an observational correlation arises. Genetic research has made enormous progress over the last years, and now provides unique opportunities to investigate shared risk and causality between traits applying new statistical tools and results from genome-wide association studies (GWAS). In the present study, we leveraged on results from the largest GWAS on 25-OH-D (79 366 samples), ${ }^{14}$ n3-PUFA (24925 samples) ${ }^{19}$, and MDD (135 458 cases and 344901 controls). ${ }^{15}$ We estimated the degree of genetic overlap between traits using both individual-level data (in NESDA) and summary-level data analyses combining GWAS summary statistics. Using summary data, Mendelian randomization analyses explored the potential bidirectional causality between 25OH-D, n3-PUFA, and MDD.

\section{Methods}

\section{Target sample for individual-level data analyses}

Individual-level data analyses were based on 2047 unrelated participants $(67.2 \%$ females $)$ of European ancestry from the NESDA. Detailed descriptions of the rationale, design, and methods for the study are given elsewhere. ${ }^{7,8}$ Briefly, NESDA is an ongoing cohort study into the long-term course and consequences of depressive and anxiety disorders. In 2004-2007, 2981 participants aged 18-65 years were recruited from the community (19\%), general practice (54\%), and secondary mental health care (27\%), and were followed-up during biannual assessments. The research protocol was approved by the ethical committee of participating universities, and all respondents provided written informed consent.

Genotyping, quality control, and imputation were previously described in detail ${ }^{20}$ (see also Supplementary Material s1). Genotype data were used to build a relationship matrix measuring genetic similarity, which was pruned at 0.05 threshold in order to retain unrelated participants.

As previously reported, ${ }^{7,8,20}$ the presence of DSM-IV (Diagnostic and Statistical Manual of Mental Disorders, 4th edition) lifetime diagnosis of MDD was assessed using the Composite Interview Diagnostic Instrument version 2.1, WHO) administered by specially trained research staff at baseline, 2-year and 4-year follow-up. Healthy controls included screened participants without any lifetime psychiatric disorder. Depressive symptoms severity was measured (baseline, 1-year, 2-year, and 4-year follow-up) using the 30-item self-report Inventory of Depressive Symptoms (IDS-SR $\left.{ }_{30}\right) \cdot{ }^{21}$ For the present analyses, scores across assessments were averaged in order to index the participant's stable exposure to depressive symptoms.

Concentrations of 25-OH-D $(N=2013)$ and n3-PUFA $(N=2010)$ were quantified in blood samples taken at baseline as previously described. ${ }^{7,8}$ Serum 25-OH-D was 
measured using isotope dilution-online solid-phase extraction liquid chromatography-tandem mass spectrometry. n3-PUFA was included in a metabolite panel measured using a high-throughput proton nuclear magnetic resonance (NMR) platform (Nightingale Health Ltd., Helsinki, Finland).

Differences in main characteristics between participants with MDD and healthy controls were analyzed with $\chi^{2}$ or $t$ test, as appropriate. The sex-adjusted association of 25$\mathrm{OH}-\mathrm{D}$ and n3-PUFA with MDD and IDS-SR ${ }_{30}$ scores was estimated using logistic and linear regression models. All analyses were performed in R v.3.5.2 (R Project for Statistical Computing).

\section{GWAS summary statistics}

Summary statistics were obtained from large GWAS of international consortia. The SUNLIGHT consortium ${ }^{14}$ performed a GWAS on circulating concentrations of 25OH-D from in 79366 samples. Summary statistics for n3PUFA were obtained from the MAGNETIC NMR GWAS $^{19}$ examining the same metabolomics platform adopted in NESDA on up to 24925 individuals. The Psychiatric Genomics Consortium (PGC) ${ }^{15}$ performed an overarching meta-analysis ${ }^{2}$ of all available GWAS datasets with MDD, including 135458 cases and 344901 controls.

\section{Polygenic risk scores}

Polygenic risk scores (PRS) were calculated as the number of risk alleles weighted by their effect sizes from the relative discovery GWAS summary statistics. PRS for 25-OH-D and n3-PUFA were based on genome-wide significant SNPs, since the genetic architecture of these traits may be characterized by few biologically relevant loci, harboring relevant genes for their synthesis and metabolism. For 25-OH-D we selected the six independent SNPs reported in the discovery GWAS. ${ }^{14}$ For n3-PUFAs, we selected seven independent genome-wide significant SNPs by processing discovery GWAS summary statistics (Supplementary Materials s2, Supplementary eTable 1). The top SNP was located in the $3^{\prime}-$ UTR region of FADS1 (fatty acid desaturase enzyme, involved in fatty acids metabolism), confirming the biological relevance of the top GWAS hits for this trait. PRS for MDD was built based on the full polygenic signal from the MDD GWAS according to LDpred method, ${ }^{22}$ which has shown an improved predictive performance compared with other methods by modeling a priori on effect sizes and LD information. In additional analyses, we recalculated the PRS for 25-OH-D and n3PUFA using the full polygenic signal of the relative discovery GWAS instead of selecting only genome-wide significant SNPs. The new PRS was built using LDpred ${ }^{22}$ with the same protocol used for MDD PRS and assuming an infinitesimal model. All PRS were standardized to aid comparison and interpretation of the results. For all details of PRS calculation see Supplementary Materials s3.1.

\section{PRS analyses}

Same- and cross-trait associations of the different PRS with 25-OH-D and n3-PUFA concentrations and with MDD diagnosis were estimated using regression models (linear for 25-OH-D and n3-PUFA and binary logistic for MDD) adjusted for sex and 10 ancestry-informative genetic principal components. The proportion of phenotypic variance explained by PRS was additionally estimated (Supplementary Materials s3.2). In additional analyses focusing on MDD cases, the association between the PRS and symptom severity measured by IDS-SR ${ }_{30}$ was also estimated adjusting for sex and principal components. All analyses were performed in R.

\section{Mendelian randomization}

Two-sample Mendelian randomization (2SMR) analyses $^{23}$ based on GWAS summary statistics were performed to test the potential causal role of 25-OH-D and n3-PUFA on MDD risk and, inversely, of MDD on 25$\mathrm{OH}-\mathrm{D}$ and n3-PUFA levels. MR infers causality by using sets of SNPs reliably associated with an exposure as instrument for this exposure and regressing SNPexposure effects against SNP-outcome effects. For each trait used as exposure, genome-wide significant independent SNPs were selected as instruments (Supplementary Materials s4.1). The use of 2SMR, as compared to classic one-sample MR, allows to significantly increase the statistical power by leveraging on effect sizes estimated in large GWAS. In order to reach an adequate power to detect a significant causal effect of 25-OH-D on MDD and of n3-PUFA on MDD in one-sample MR, the number of MDD cases needed (coupled with the same number of controls) would be, respectively, $\sim 8000$ and $\sim 100000$ (Supplementary Materials s4.2). 2SMR analyses were based on inverse-variance-weighted fixed-effects metaanalysis, pooling test statistics of all SNPs, inversely weighted by their standard error. Main sensitivity analyses were based on weighted-median ${ }^{24}$ and weighted-mode ${ }^{25}$ causal estimators, providing consistent causal estimates even when the majority of instruments are violating MR assumptions. Appropriate analytical checks of heterogeneity were additionally performed, including Cochran's $Q$ test, single SNP, and leave-one-out SNP analyses. Additional analyses examined the potential impact on results of using weak genetic instruments explaining a limited amount of exposures variance. Polygenic instruments including larger sets of SNPs were built for each exposure following the same procedures described in Supplementary Materials s4.1, but applying a more lenient threshold for inclusion based on an SNP-trait association $p$ 
value $<5.0 \mathrm{e}-05$ (similar threshold adopted by Richardson et $\mathrm{al}^{26}$ ) in the discovery GWAS. The new instruments were used in 2SMR analyses robust against pleiotropy-related violations potentially induced by the introduction of a large number of SNPs, including MR-Egger and MRRAPS (robust adjust profile scores), ${ }^{27}$ a recently proposed method that could provide unbiased estimates in the presence of many weak variants. All analyses were conducted using the MR-Base R package. ${ }^{28}$

\section{Results}

\section{Individual-level data analyses: descriptives}

The NESDA dataset included 1700 participants with a diagnosis of lifetime MDD and 347 healthy controls. MDD cases, as compared to controls, were more likely to be females and had lower blood levels of 25-OH-D (Cohen's $d-0.25$ ) and n3-PUFA (Cohen's $d-0.15$ ) (Table 1). The odds of lifetime MDD was reduced by $\sim 20 \%$ for each SD increase in 25-OH-D levels (sexadjusted $\mathrm{OR}=0.78,95 \%$ confidence intervals $(\mathrm{CIs})=$ $0.70-0.87, p=1.4 \mathrm{e}-5)$ and by $\sim 10 \%$ for each SD increase in n3-PUFA levels (sex-adjusted OR $=0.87,95 \%$ $\mathrm{CIs}=0.78-0.97, p=1.1 \mathrm{e}-2$ ). Sex-adjusted levels of 25$\mathrm{OH}-\mathrm{D}$ and n3-PUFA were associated with depressive symptoms severity (Fig. 1). Levels of 25-OH-D were inversely and linearly related to IDS-SR 30 scores, while the association between n3-PUFA and symptom severity was best approximated by a quadratic form indicating higher depressive symptoms, especially below a certain level of n3-PUFA.

\section{Individual-level data analyses: PRS analyses}

Table 2 shows same- and cross-trait associations of the PRS with 25-OH-D and n3-PUFA concentrations and with MDD. The association between PRS and phenotypes of the same trait were highly significant (PRS 25-OH-D

Table 1 Characteristics of the target sample (NESDA) for individual-level data analyses

\begin{tabular}{llll}
\hline Characteristics & $\begin{array}{l}\text { MDD cases } \\
(\boldsymbol{N}=\mathbf{1 7 0 0})\end{array}$ & $\begin{array}{l}\text { Controls } \\
(\boldsymbol{N}=\mathbf{3 4 7})\end{array}$ & $\boldsymbol{p}$ Value \\
\hline Age (years) (mean $\pm \mathrm{SD})$ & $42.4(12.5)$ & $43.5(14.2)$ & 0.17 \\
Sex (F) (\%) & 68.6 & 60.1 & $4.3 \mathrm{E}-03$ \\
Average IDS-SR 30 & $21.7(11.5)$ & $6.2(5.0)$ & $2.6 \mathrm{E}-225$ \\
$\begin{array}{l}\text { (mean } \pm \text { SD) } \\
\text { 25-OH-D (nmol/L) }\end{array}$ & $63.7(28.3)$ & $70.5(26.7)$ & $4.8 \mathrm{E}-05$ \\
(mean \pm SD) & & & \\
n3-PUFAs (nmol/L) & $0.38(0.13)$ & $0.40(0.14)$ & $1.6 \mathrm{E}-02$ \\
(mean \pm SD) & & & \\
\hline
\end{tabular}

Unit of measure $25-\mathrm{OH}-\mathrm{D}$, n3-PUFA: $\mathrm{nmol} / \mathrm{L}$ (multiply by 0.4 to obtain $\mathrm{ng} / \mathrm{mL}$ ) $p=1.4 \mathrm{e}-20$, PRS n3-PUFA $p=9.3 \mathrm{e}-6$, PRS MDD $p=1.4 \mathrm{e}-4)$. The respective PRS explained $3.5 \%$ of trait variance for $25-\mathrm{OH}-\mathrm{D}$ level, $0.8 \%$ for n3-PUFA levels, and $1.5 \%$ of MDD liability variance. Nevertheless, no association was found between PRS of one trait and the other phenotypes, suggesting a lack of shared genetic effects across traits.

Consistent with these results, analyses focusing on lifetime MDD cases showed that only the PRS for MDD was associated with higher average 4-year severity of depressive symptom, explaining $0.7 \%$ of their variance (Fig. 2). PRS for 25-OH-D and n3-PUFA were not related to symptoms severity in MDD cases.

In additional analyses, we recalculated the PRS for 25$\mathrm{OH}-\mathrm{D}$ and n3-PUFA using the full polygenic signal emerging from the discovery GWAS ${ }^{14,19}$ instead of selecting only genome-wide significant SNPs. The resulting PRS were less strongly, although significantly, associated with the relative phenotype (25-OH-D $\beta=$ 1.71, 95\% CIs $=0.59-2.84, p=2.9 \mathrm{e}-3$; n3-PUFA $\beta=$ $0.01,95 \% \mathrm{CIs}=0.002-0.01, p=9.9 \mathrm{e}-3)$, but explained a smaller proportion of variance $(0.4 \%$ for $25-\mathrm{OH}-\mathrm{D}$ and $0.3 \%$ for n3-PUFA) as compared to the PRS based on the top SNPs, confirming that in the genetic architecture of 25-OH-D and n3-PUFA few biologically relevant loci may play a relative major role.

\section{Summary-level data analyses: Mendelian randomization}

Table 3 reports the main results from 2SMR analyses (Supplementary Materials s4.3 includes Supplementary eFigs. 1-4 depicting 2SMR plots). Estimates from inversevariance-weighted fixed-effects-meta-analysis indicated that the genetic instrument for 25-OH-D and n3-PUFA were not causally related to MDD risk. Of interest, only in the analyses focusing on n3-PUFA as exposure the Cochran's $Q$ heterogeneity test was significant $(p=1.5 \mathrm{e}-3)$, indicating the presence of at least one outlier SNP potentially exhibiting horizontal pleiotropy. Inspection of the plots (Supplementary eFig. 2) highlighted rs174546 (3'-UTR region of FADS1) as potential source of heterogeneity. In order to evaluate whether heterogeneity was indicating potential horizontal pleiotropy (the instrument/SNP is associated to the outcome through pathways others than the exposure), we performed a PheWAS (phenome-wide association scan, Supplementary Materials s4.4 and Supplementary eFig. 5), confirming that the SNP was significantly associated with a wide array of traits beyond $\mathrm{n} 3-$ PUFA, including for instance metabolic (e.g., cholesterol, triglycerides), cardiovascular (e.g., heart rate), or psychiatric (e.g., sleep duration, irritability), which are, in turn, associated with depression. Nevertheless, sensitivity MR analyses based on weighted-median ${ }^{24}$ and weightedmode $^{25}$ causal estimators, providing consistent causal estimates even when the majority of instruments are 


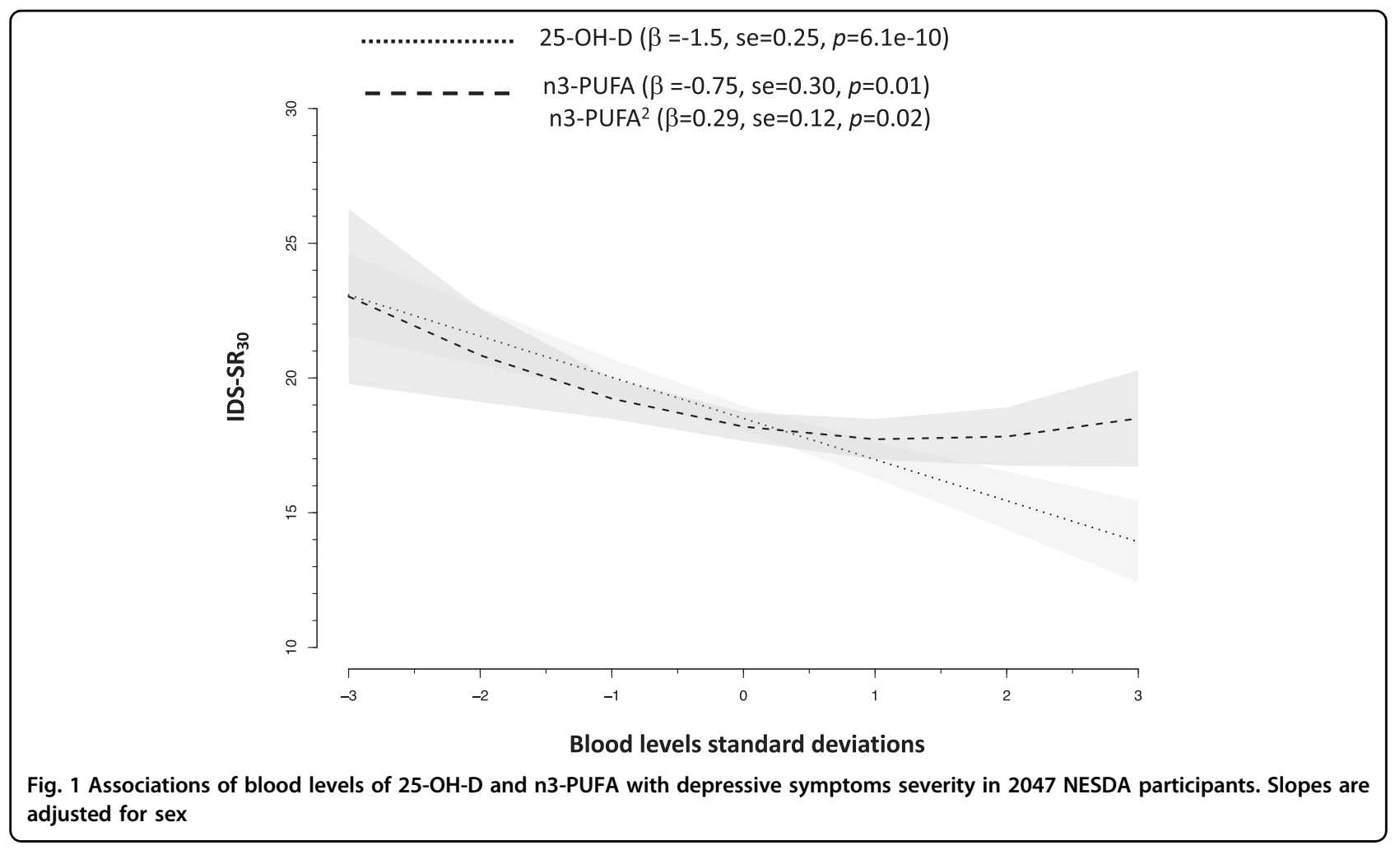

Table 2 Same- and cross-trait associations of polygenic risk scores with circulating 25-hydroxyvitamin D, omega-3 fatty acids, and major depressive disorder in >2000 participants from NESDA

\begin{tabular}{|c|c|c|c|c|c|c|c|c|c|}
\hline \multirow[b]{3}{*}{ PRS } & \multicolumn{3}{|l|}{ 25-OH-D } & \multicolumn{3}{|l|}{ n3-PUFA } & \multirow{2}{*}{\multicolumn{3}{|c|}{$\frac{\text { MDD }}{1700 \text { cases }+347 \text { controls }}$}} \\
\hline & \multirow{2}{*}{$\begin{array}{l}N=2013 \\
\beta\end{array}$} & \multicolumn{2}{|c|}{ Mean $=64.8(28.2) \mathrm{nmol} / \mathrm{L}$} & \multirow{2}{*}{$\begin{array}{l}N=2010 \\
\beta\end{array}$} & \multicolumn{2}{|c|}{ Mean $=0.39(0.13) \mathrm{nmol} / \mathrm{L}$} & & & \\
\hline & & $95 \% \mathrm{Cls}$ & $p$ Value & & $95 \% \mathrm{Cls}$ & $p$ Value & OR & $95 \% \mathrm{Cls}$ & $p$ Value \\
\hline 25-OH-D & 5.29 & $4.18-6.39$ & $1.4 \mathrm{E}-20$ & -0.001 & -0.01 to 0.004 & 0.74 & 0.94 & $0.84-1.06$ & 0.34 \\
\hline n3-PUFA & -0.49 & -1.61 to 0.63 & 0.39 & 0.01 & $0.007-0.02$ & $9.3 E-06$ & 1.01 & $0.90-1.14$ & 0.83 \\
\hline MDD & -0.57 & -1.70 to 0.55 & 0.32 & 0.001 & -0.03 to 0.01 & 0.58 & 1.26 & $1.12-1.41$ & $1.4 \mathrm{E}-04$ \\
\hline
\end{tabular}

Results from linear (outcome: 25-OH-D, n3-PUFA) and binary logistic (outcome: MDD) regression analyses adjusted for sex and ten ancestry-informative genetic principal components

Unit of measure $25-\mathrm{OH}-\mathrm{D}, \mathrm{n3}-\mathrm{PUFA}$ : $\mathrm{nmol} / \mathrm{L}$ (multiply by 0.4 to obtain $\mathrm{ng} / \mathrm{mL}$ )

Bold values indicates statistically significant $p<0.05$

violating MR assumptions, confirmed that 25-OH-D and n3-PUFA levels were not causal for MDD.

In the opposite direction, 2SMR analyses examining MDD as exposure consistently showed no significant evidence of a causal role on 25-OH-D and n3-PUFA levels. Additionally, analyses using MR-Egger method ${ }^{29}$ were used (Supplementary Material s4.5) and results are presented in Supplementary eTable 2. Although the number of instruments used to test the causal effect of 25-OH-D and n3PUFA was lower than that $(N=10)$ recommended to run an adequately powered MR-Egger analyses, the estimates were substantially consistent with those obtained by other analyses, confirming the lack of potential causal effect of 25-OH-D and n3-PUFA on MDD and vice versa.

Additional analyses examined the potential impact of low statistical power due to weak instruments explaining a limited amount of exposures variance, in particular those indexing MDD. For instance, a PRS built with the 37 SNPs used as instruments in MDD on 25-OH-D 2SMR analyses was weakly associated with lifetime MDD in NESDA (sex- and PC-adjusted association between the PRS and MDD, per SD increase: odds ratio $(\mathrm{OR})=1.13,95 \% \mathrm{CIs}=1.01-1.26, p=3.7 \mathrm{e}-2)$ and accounted only for $0.4 \%$ of its liability. New instruments 


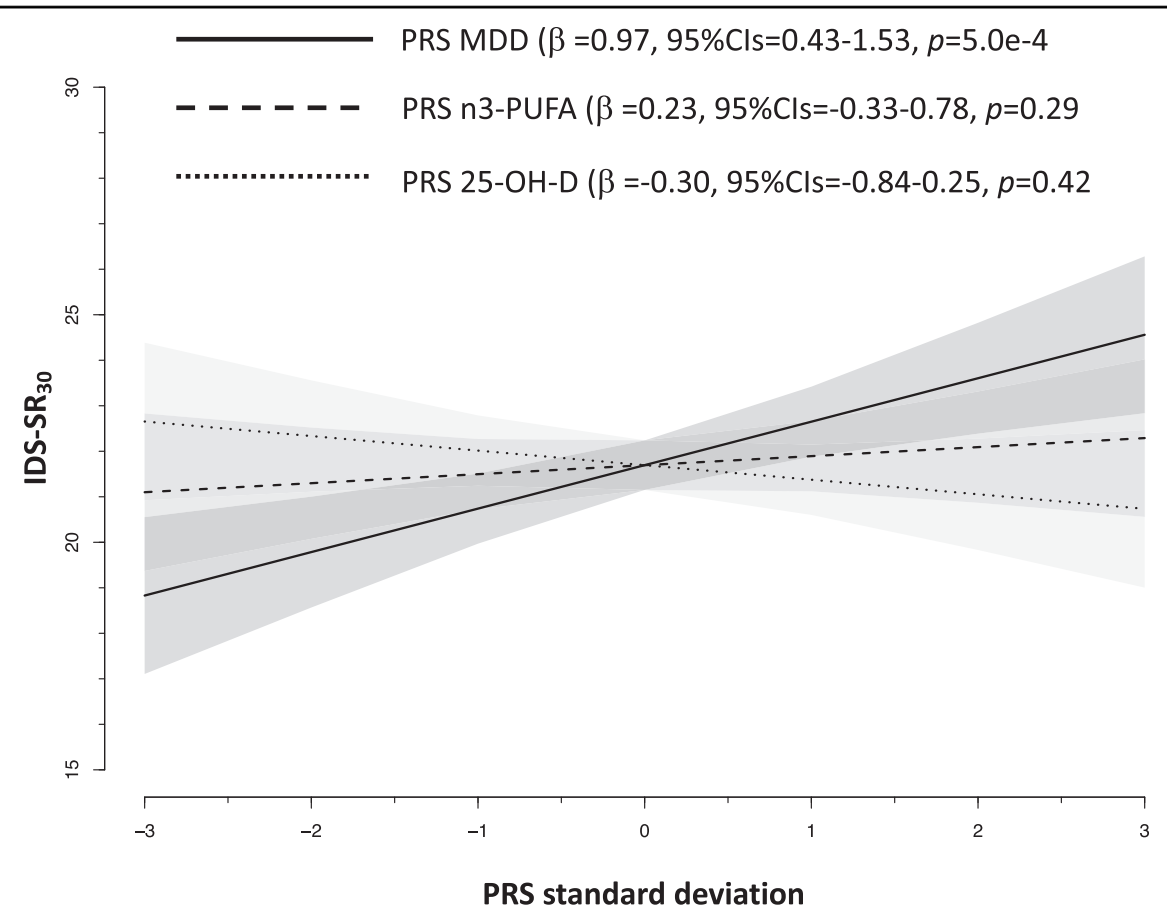

Fig. 2 Associations between polygenic risk scores and depressive symptoms severity in 1700 cases with major depressive disorder from NESDA. Slopes are adjusted for sex and 10 ancestry-informative principal components

Table 3 Two-samples Mendelian randomization analyses based on GWAS summary statistics estimating causal effects between circulating 25-hydroxyvitamin D, omega-3 fatty acids, and major depressive disorder

\begin{tabular}{|c|c|c|c|c|c|c|c|c|c|c|c|}
\hline \multirow[t]{2}{*}{ Exposure } & \multirow[t]{2}{*}{ Outcome } & \multirow[t]{2}{*}{$N$ SNPs } & \multicolumn{3}{|c|}{ Inverse-variance weighted } & \multicolumn{3}{|c|}{ Weighted median } & \multicolumn{3}{|c|}{ Weighted mode } \\
\hline & & & Estimate & SE & $p$ Value & Estimate & SE & $p$ Value & Estimate & SE & $p$ Value \\
\hline $25-\mathrm{OH}-\mathrm{D}$ & MDD & 6 & -0.03 & 0.05 & 0.50 & -0.02 & 0.06 & 0.66 & -0.02 & 0.06 & 0.73 \\
\hline n3-PUFA & MDD & 7 & -0.06 & 0.04 & 0.16 & -0.05 & 0.04 & 0.13 & -0.08 & 0.04 & 0.12 \\
\hline MDD & 25-OH-D & 37 & -0.02 & 0.01 & 0.25 & -0.002 & 0.02 & 0.90 & 0.01 & 0.03 & 0.66 \\
\hline MDD & n3-PUFA & 44 & 0.03 & 0.07 & 0.66 & 0.02 & 0.09 & 0.82 & -0.08 & 0.20 & 0.71 \\
\hline
\end{tabular}

Units of measure in discovery GWAS: 25-OH-D, 1 unit increase in (log) concentrations; n3-PUFA, 1 unit increase in (inverse normal transformation) concentrations; MDD, 1 log-unit increase in risk

SNP effects heterogeneity assessment in IVW: 25-OH-D causal for MDD, Cochran's $Q p=0.79$; n3-PUFA causal for 25-OH-D, Cochran's $Q p=1.5 \mathrm{e}-3$; MDD causal for 25-OH-D, Cochran's $Q p=0.15$; MDD causal for n3-PUFA, Cochran's $Q p=0.17$

including larger sets of SNPs were built (25-OH-D: 132 SNPs; n3-PUFA, 132 SNPs; MDD on 25-OH-D: 477 SNPs; MDD on n3-PUFA: 675 SNPs). Results from MREgger and MR-RAPS analyses confirmed the lack of significant causal estimates (Supplementary eTable 3), suggesting that the original findings were not hampered by weak instruments.

Finally, analyses based on quantitative genetics theory (Supplementary Materials s5 and Supplementary eTable 4) further strengthened the conclusion of a lack of causal relationships between 25-OH-D, n3-PUFA, and MDD.

\section{Discussion}

In the present study, we examined the nature of the association of 25-OH-D and n3-PUFA with MDD using the latest data and analytical tools developed in genomics. We found no significant evidence of shared genetic risk or direct causality between vitamin D or n3-PUFA and MDD; unresolved confounding ${ }^{13}$ should be considered at 
this stage, the most likely explanation for the association reported by observational studies.

Evidence of a common genetic base consistently shared between traits was not found. Even in data from the NESDA cohort, showing phenotypic associations of 25$\mathrm{OH}-\mathrm{D}$ and $\mathrm{n} 3-\mathrm{PUFA}$ levels with MDD diagnosis presence and severity of depressive symptoms, ${ }^{7,8}$ the underlying polygenic risk for each trait was not associated with the phenotype of the others, indicating the lack of genetic overlap. Statistically non-significant genome-wide genetic correlations between 25-OH-D and MDD and n3-PUFA and MDD were previously reported. ${ }^{14,15}$ Nevertheless, those results may have been affected by the use of data for depression from GWAS relatively underpowered as compared to those available in the present study, and by different genetic architectures of MDD (highly polygenic) vs. 25-OH-D or n3-PUFA (few genetic loci with relatively larger effect sizes). In the present analyses, despite the use of polygenic scores for 25-OH-D or n3-PUFA built only on GWAS significant loci, including biologically relevant genes for their synthesis and metabolism, no cross-trait association was found, suggesting that the biological pathways encompassing those genes are not shared with depression.

Furthermore, different MR analyses provided no evidence of a direct causal effect of 25-OH-D or n3-PUFA levels on MDD risk, despite the use of strong genetic instruments and the use of 2SMR techniques leveraging data from large GWAS studies. Furthermore, a potential causal effect of MDD on 25-OH-D or n3-PUFA levels was not detected, suggesting that also reverse causation does not explain the phenotypic association between these traits.

The present findings are consistent with other emerging evidence. A concomitant analysis ${ }^{30}$ applying MR techniques sustains the lack of causal effect of 25-OH-D on MDD, although the study was limited by the use of data for depression from a smaller GWAS ( one-third of the cases available here) and the lack of testing the potential reverse effect of MDD on 25-OH-D. Furthermore, a first study $^{31}$ applying MR in a single (low-powered) pregnancy cohort found no evidence of a significant causal effect of n3-PUFA on antenatal, perinatal, and postnatal depression.

Findings from the present study suggest that the observational associations of vitamin D or n3-PUFA and MDD, commonly reported in epidemiological studies, may be likely explained by unresolved confounding. The relationship of blood levels of 25-OH-D and n3-PUFA with MDD may indeed be confounded by several factors, such as health-related lifestyles (e.g., physical activity, diet), personality traits (e.g., conscientiousness and neuroticism), and comorbidity (e.g., obesity). ${ }^{32,33}$ Interestingly, we showed here that the top genetic variant linked to n3-PUFA levels on the FADS1 gene is also associated with a wide array of traits, among which metabolic (e.g., cholesterol, triglycerides), cardiovascular (e.g., heart rate), and psychiatric (e.g., sleep duration, irritability) features that are, in turn, associated with MDD. ${ }^{18}$ In previous results from $\mathrm{NESDA}^{7,8}$, we showed that adjustment for available major sociodemographic, lifestyle, and health confounders substantially reduced the effect size, although remaining statistically significant, of the association between depression and 25-OH-D or n3-PUFA. Nevertheless, not all key confounders could be exhaustively measured, and while some were not available (e.g., dietary intake) other very important ones, such as actual exposure to sunlight impacting on 25-OH-D levels, could be only approximated by imperfect proxies.

Despite the fact that the relationship of MDD with 25OH-D or n2-PUFA may be confounded and not be direct, epidemiological evidence consistently showing that depressed patients have lower blood level of these biological markers still raises an important concern for other health problems. For instance, we previously reported ${ }^{7}$ that one-third of patients with established psychiatric diagnoses of depressive disorders had 25-OH-D blood concentrations considered insufficient and a risk for musculoskeletal complications. Normalization of vitamin $\mathrm{D}$ in depressed patients may therefore be important to prevent related complications and disability, in particular during late life.

A major strength of the present study is the application of the latest techniques developed in genetic epidemiology, with analyses performed both at the individual-level data, using information from a large and psychiatrically well-characterized cohort, and at the summary-level data, combining GWAS summary statistics from the largest international consortia. An important limitation considering MR results is that genetic variants index the average lifetime exposure to risk factors and consequently could estimate an average lifetime causal effect without being able to identify a specific time in life in which the causal effect may take place. For instance, MR studies ${ }^{34}$ on multiple sclerosis suggested the existence of sensitive periods for the protective effect of vitamin D. Nevertheless, epidemiological data show that the association of 25-OH-D and n3-PUFA with MDD risk is consistent across different ranges of ages. ${ }^{3,4,7,8}$ Furthermore, the lack of statistically significant causal estimates, especially in analyses considering MDD as an exposure, may have resulted from low statistical power due to weak genetic instruments. Nevertheless, sensitivity analyses with polygenic instruments including larger sets of SNPs confirmed the results of original analyses. In the future, results from larger GWAS, as compared to those currently available, could be used to further confirm the present findings. Another major limitation is that both individual- and 
summary-level data analyses were based on samples of European ancestry and therefore results cannot be generalized to different populations. Finally, a major limitations is represented by the heterogeneity of the MDD diagnoses, which may aggregate different sub-disorders characterized by distinct pathophysiology. We previously demonstrated $^{35,36}$ that MDD patients could be divided based on symptom profiles in subgroups characterized by partially distinct polygenic signatures. It could be hypothesized that 25-OH-D and n3-PUFA may have a causal role only in specific subtype of MDD. Nevertheless, large GWAS are needed on potential MDD subtypes, yet unavailable, in order to be used for adequately powered MR analyses.

Depression-related disability is a major health and economic burden for societies that require an urgent answer. Nevertheless, despite depression's public health relevance, treatment options available nowadays are not optimal in terms of efficacy. Epidemiological evidences are constantly scanned in order to identify potentially actionable target that may substantially improve the prevention and treatment of depression. Recently developed genomic tools could be efficiently employed to examine the nature of observational associations emerging in epidemiology, providing some indications on the most promising associations to be prioritized in subsequent intervention studies. The premature and direct translation of observational associations in nutritional epidemiology into supplementation interventions may encounter indeed disappointing results, as emerging for different health outcomes. For instance, recent large-scale meta-analyses of randomized control studies have shown, for instance, that vitamin D supplementation does not prevent fractures, falls, or improve bone mineral density, ${ }^{37}$ or that n3-PUFA supplementation had no significant effect on coronary heart disease or major vascular events. ${ }^{38}$ More recently, results from the large VITAL $^{39}$ trial testing n3-PUFA supplementation in $>25000$ participants showed no significant impact on the primary prevention of cardiovascular diseases or cancer.

In conclusion, we believe that findings from the present study, in conjunction with previous conflicting evidence from clinical studies, represent a cautionary tale for further research testing the potential therapeutic effect of vitamin D and n3-PUFA supplementation on depression, as the expectations of a direct causal effect of these compounds on mood should be substantially reconsidered. Upcoming results from already established largescale randomized controlled trials such as MoodFOOD ${ }^{1}$ or VITAL-DEP ${ }^{2}$ will tell whether this conclusion is correct. During the evaluation of the present manuscript results for the MoodFOOD trial were published, ${ }^{40}$ confirming that multinutrient supplementation (including vitamin D and omega-3) was not effective in preventing the onset of major depressive episodes.

\section{Acknowledgements}

For NESDA, funding was obtained from the Netherlands Organization for Scientific Research (Geestkracht program grant 10-000-1002); the Center for Medical Systems Biology (CSMB, NWO Genomics), Biobanking and

Biomolecular Resources Research Infrastructure (BBMRI-NL), VU University's Institutes for Health and Care Research (EMGO+) and Neuroscience Campus Amsterdam, University Medical Center Groningen, Leiden University Medical Center, National Institutes of Health (NIH, R01D0042157-01A, MH081802, Grand Opportunity grants 1RC2 MH089951 and 1RC2 MH089995). Part of the genotyping and analyses were funded by the Genetic Association Information Network (GAIN) of the Foundation for the National Institutes of Health. Computing was supported by BiG Grid, the Dutch e-Science Grid, which is financially supported by NWO. We would like to thank the investigators from the PGC, the SUNLIGHT Consortium, and the MAGNETIC NMR GWAS Consortium for sharing summary statistics of their GWAS. In order to perform the present analyses, we received summary statistics of the GWAS by Hyde et al. (Nat. Genet. 2016;48:1031-6) from 23andMe. We would also like to thank research participants and employees of 23andMe for making this work possible. WJP was funded by an NWO Veni grant (91619152).

\section{Author details}

'Department of Psychiatry, Amsterdam Public Health and Amsterdam Neuroscience, Amsterdam UMC, Vrije Universiteit/GGZ inGeest, Amsterdam, Netherlands. ${ }^{2}$ Department of Biological Psychology, VU University Amsterdam, Amsterdam, Netherlands. ${ }^{3}$ Qatar Genome Programme, Qatar Foundation, Doha, Qatar

\section{Conflict of interest}

B.W.J.H.P. has received research funding (non-related to the work reported here) from Jansen Research and Boehringer Ingelheim. The other authors declare that they have no conflict of interest.

\section{Publisher's note}

Springer Nature remains neutral with regard to jurisdictional claims in published maps and institutional affiliations.

Supplementary Information accompanies this paper at (https://doi.org/ 10.1038/s41398-019-0554-y).

Received: 22 March 2019 Revised: 21 May 2019 Accepted: 20 June 2019 Published online: 05 September 2019

\section{References}

1. Roca, M. et al. Prevention of depression through nutritional strategies in highrisk persons: rationale and design of the MooDFOOD prevention trial. BMC Psychiatry 16, 192 (2016)

2. Okereke, O. I. et al. The VITamin D and OmegA-3 TriaL-Depression Endpoint Prevention (VITAL-DEP): rationale and design of a large-scale ancillary study evaluating vitamin $D$ and marine omega-3 fatty acid supplements for prevention of late-life depression. Contemp. Clin. Trials 68, 133-145 (2018).

3. Anglin, R. E. S., Samaan, Z., Walter, S. D. \& McDonald, S. D. Vitamin D deficiency and depression in adults: systematic review and meta-analysis. Br. J. Psychiatry 202, 100-107 (2013).

4. Lin, P. Y., Huang, S. Y. \& Su, K. P. A meta-analytic review of polyunsaturated fatty acid compositions in patients with depression. Biol. Psychiatry 68, 140-147 (2010).

5. Okereke, O. I. \& Singh, A. The role of vitamin D in the prevention of late-life depression. J. Affect Disord. 198, 1-14 (2016).

6. Smith, M., Beilin, L. \& Mori, T. Essential fatty acids and mood: a systematic review of observational studies. Am. J. Food Nutr. 1, 14-27 (2011).

7. Milaneschi, Y. et al. The association between low vitamin $D$ and depressive disorders. Mol. Psychiatry 1936, 444-451 (2014). 
8. Thesing, C. S., Bot, M., Milaneschi, Y. \& Giltay, E. J. Penninx BWJH. Omega-3 and omega- 6 fatty acid levels in depressive and anxiety disorders. Psychoneuroendocrinology 87, 53-62 (2018).

9. Gowda, U., Mutowo, M. P., Smith, B. J., Wluka, A. E. \& Renzaho, A. M. N. Vitamin D supplementation to reduce depression in adults: meta-analysis of randomized controlled trials. Nutrition 31, 421-429 (2015).

10. Spedding, S. Vitamin D and depression: a systematic review and meta-analysis comparing studies with and without biological flaws. Nutrients 6, 1501-1518 (2014).

11. Mocking, R. J. T. et al. Meta-analysis and meta-regression of omega-3 polyunsaturated fatty acid supplementation for major depressive disorder. Transl. Psychiatry 6, e756 (2016).

12. Bloch, M. H. \& Hannestad, J. Omega-3 fatty acids for the treatment of depression: systematic review and meta-analysis. Mol. Psychiatry 17, 1272-1282 (2012).

13. loannidis, J. P. A. The challenge of reforming nutritional epidemiologic research. JAMA 320, 969-970 (2018). 11.

14. Jiang, X. et al. Genome-wide association study in 79,366 European-ancestry individuals informs the genetic architecture of 25-hydroxyvitamin D levels. Nat. Commun. 9, 260 (2018)

15. Major Depressive Disorder Working Group of the Psychiatric Genomics Consortium. Genome-wide association analyses identify 44 risk variants and refine the genetic architecture of major depressive disorder. Nat. Genet. 50, 668-681 (2018).

16. McCann, J. \& Ames, B. Is there convincing biological or behavioral evidence linking vitamin D deficiency to brain dysfunction? FASEB J. 22, 982-1001 (2008).

17. Su, K. P., Matsuoka, Y. \& Pae, C. U. Omega-3 polyunsaturated fatty acids in prevention of mood and anxiety disorders. Clin. Psychopharmacol. Neurosci. 13, 129-137 (2015).

18. Otte, C. et al. Major depressive disorder. Nat. Rev. Dis. Primers 2, 16065 (2016).

19. Kettunen, J. et al. Genome-wide study for circulating metabolites identifies 62 loci and reveals novel systemic effects of LPA. Nat. Commun. 7, 11122 (2016).

20. Mbarek, H. et al. Genome-wide significance for PCLO as a gene for major depressive disorder. Twin Res. Hum. Genet. 20, 267-270 (2017).

21. Rush, A., Gullion, C., Basco, M., Jarrett, R. \& Trivedi, M. The inventory of depressive symptomatology (IDS): psychometric properties. Psychol. Med. 26, 477-486 (1996).

22. Vilhjálmsson, B. J. et al. Modeling linkage disequilibrium increases accuracy of polygenic risk scores. Am. J. Hum. Genet. 97, 576-592 (2015).

23. Davey Smith, G. \& Hemani, G. Mendelian randomization: genetic anchors for causal inference in epidemiological studies. Hum. Mol. Genet. 23, R89-R98 (2014).

24. Bowden, J., Davey Smith, G., Haycock, P. C. \& Burgess, S. Consistent estimation in Mendelian randomization with some invalid instruments using a weighted median estimator. Genet. Epidemiol. 40, 304-314 (2016).
25. Hartwig, F. P., Davey Smith, G. \& Bowden, J. Robust inference in summary data Mendelian randomization via the zero modal pleiotropy assumption. Int. J. Epidemiol. 46, 1985-1998 (2017).

26. Richardson, T. G., Harrison, S., Hemani, G. \& Smith, G. D. An atlas of polygenic risk score associations to highlight putative causal relationships across the human phenome. Elife 5, e43657 (2019).

27. Zhao, Q., Wang, J., Hemani, G., Bowden, J. \& Small D. S. Statistical inference in two-sample summary-data Mendelian randomization using robust adjusted profile score. Preprint at http://arxiv.org/abs/1801.09652 (2019).

28. Hemani, G. et al. The MR-Base platform supports systematic causal inference across the human phenome. Elife 7, e34408 (2018).

29. Bowden, J., Smith, G. D. \& Burgess, S. Mendelian randomization with invalid instruments: effect estimation and bias detection through Egger regression. Int. J. Epidemiol. 44, 512-525 (2015).

30. Michaëlsson, K., Melhus, H. \& Larsson, S. Serum 25-hydroxyvitamin D concentrations and major depression: a Mendelian randomization study. Nutrients 10, 1987 (2018).

31. Sallis, H., Steer, C., Paternoster, L., Davey Smith, G. \& Evans, J. Perinatal depression and omega-3 fatty acids: a Mendelian randomisation study. J. Affect Disord. 166, 124-131 (2014).

32. Van Dam, R. M. et al. Potentially modifiable determinants of vitamin D status in an older population in the Netherlands: the Hoorn study. Am. J. Clin. Nutr. 85 755-761 (2007)

33. Thesing, C. S., Bot, M., Milaneschi, Y., Giltay, E. J. \& Penninx, B. W. J. H. The association of omega-3 fatty acid levels with personality and cognitive reactivity. J. Psychosom. Res. 108, 93-101 (2018).

34. Harroud, A. \& Richards, J. B. Mendelian randomization in multiple sclerosis: a causal role for vitamin D and obesity? Mult. Scler. 24, 80-85 (2018).

35. Milaneschi, Y. et al. Polygenic dissection of major depression clinical heterogeneity. Mol. Psychiatry 21, 516-522 (2015).

36. Milaneschi, Y. et al. Genetic association of major depression with atypical features and obesity-related immunometabolic dysregulations. JAMA Psychiatry 74, 1214-1225 (2017)

37. Bolland, M., Grey, A. \& Avenell, A. Effects of vitamin D supplementation on musculoskeletal health: a systematic review, meta-analysis, and trial sequential analysis. Lancet Diabetes Endocrinol. 6, 847-858 (2018).

38. Aung, T. et al. Associations of omega-3 fatty acid supplement use with cardiovascular disease risks meta-analysis of 10 trials involving 77917 individuals. JAMA Cardiol. 3, 225-234 (2018).

39. Manson, J. E. et al. Vitamin D supplements and prevention of cancer and cardiovascular disease. N. Engl. J. Med. https://doi.org/10.1056/NEJMoa1809944 (2018).

40. Bot, M. et al. Effect of multinutrient supplementation and food-related behavioral activation therapy on prevention of major depressive disorder among overweight or obese adults with subsyndromal depressive symptoms. The MooDFOOD Randomized Clinical Trial. JAMA $\mathbf{3 2 1}$ 858-868 (2019). 